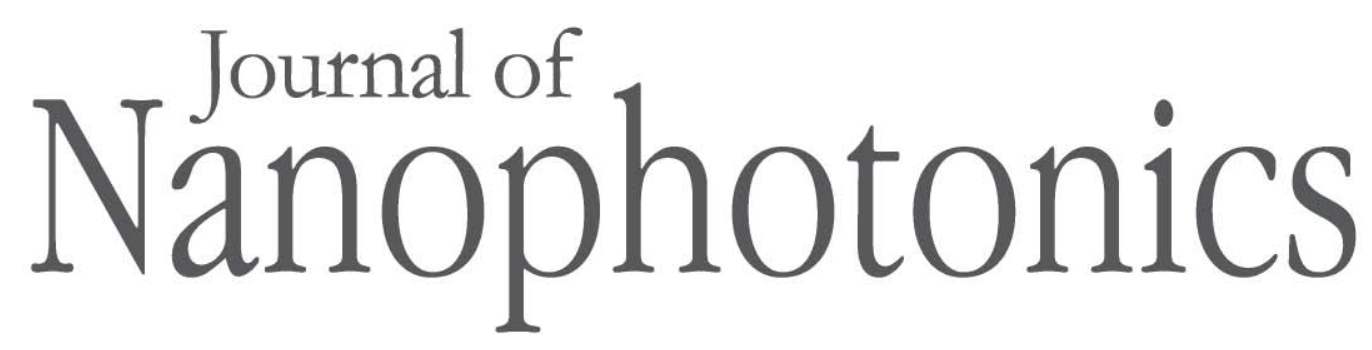

SPIEDigitalLibrary.org/jnp

\title{
Preparation of nanostructured ultrathin silver layer
}

Jiř́i Bulír

Michal Novotný

Anna Lynnykova

Ján Lančok 


\title{
Preparation of nanostructured ultrathin silver layer
}

\author{
Jiř́i Bulíř, Michal Novotný, Anna Lynnykova, and Ján Lančok \\ Institute of Physics, ASCR, v.v.i., Na Slovance 2, Prague, Czech Republic \\ bulir@fzu.cz
}

\begin{abstract}
Silver is widely used for fabrication of plasmonic devices because of its unique optical constants. The nanostructure of the Ag layer is mainly influenced during the initial stage of the silver nucleation. Therefore, we focus our attention on studying this stage of silver growth. Nanostructured ultrathin silver layers are prepared by means of magnetron sputtering. The initial stage of the nucleation and the layer growth is studied by optical monitoring, which is based on spectrophotometric measurement of the sample reflectivity. The measured data are compared to a model of layered structure. The noncontinual (Volmer-Weber) mode of the layer nucleation is clearly distinguished in the monitored data. Thus, we are able to estimate the point of noncontinual layer coalescence. The optical data are correlated with in situ monitoring of the electrical resistance. We find that the nucleation mode and resulting nanostructure can be significantly influenced by an ultrathin silver oxide interlayer. @ 2011 Society of Photo-Optical Instrumentation Engineers (SPIE). [DOI: 10.1117/1.3562568]
\end{abstract}

Keywords: ultrathin silver layers; growth monitoring; optical properties.

Paper 10072SSPR received Oct. 2, 2010; revised manuscript received Jan. 15, 2011; accepted for publication Jan. 19, 2011; published online Mar. 17, 2011.

\section{Introduction}

A considerable number of metal-dielectric devices make use of ultrathin silver layers. For instance, the typical optical metal-dielectric devices are transmission-induced filters ${ }^{1,2}$ or conductive transparent multilayer structures known as "transparent metals." ${ }^{3}$ The performance of those metal-dielectric optical devices is influenced by the quality of the metal layer and, in particular, of the metal-dielectric interface.

Complex research has been carried out in order to understand of the growth of thin metal film. ${ }^{4,5}$ The growth mode depends mainly on the wetting relation between the deposited material and the substrate. The wetting is determined by relation of the adhesion energy and the free energy of metal in a vacuum. Noble metals exhibit generally low adhesion energy to most oxides, which is caused by weak chemical bonding. These facts are a precondition for the Volmer-Weber growth mode, where isolated islands are formed at first. It is rather complicated to reach the Stranski-Krastanov growth mode, which is important for obtaining smooth and continuous thin film. Silver is known to show a tendency to noncontinuous nucleation characterized by formation of islands in the early stage of the growth.

Nanostructured materials in the form of nanocomposites, where the metallic nanoparticles are uniformly distributed in a dielectric matrix, represent a new type of materials with unique optical properties, which are quite different from those of the underlying components. Metallic nanoclusters show strong absorption bands, which are induced by excitation of the localized plasmon resonance. The enhancement of the optical absorption, which is attributed to the enhancement of the local electromagnetic field near the metal surface, can have a great impact on photoelectronic applications and in solar cell development. ${ }^{6-8}$ The resonant frequency of a localized plasmon is usually a complex function of the geometry, size, and dielectric function of

$1934-2608 / 2011 / \$ 25.00$ @ 2011 SPIE 
the metallic cluster material, and of the dielectric function of the surrounding matrix. Therefore, accurate control of the deposition process is required.

The dependence of the optical properties on the morphology of an ultrathin silver layer has been studied in a number of works. ${ }^{9-11}$ Both silver particle size and refractive index of the substrate are responsible for the redshift of the plasmon resonance peak in the absorption spectra. ${ }^{10}$ Thus, the optical spectra are very indicative of the wetting condition and growth mode. A method of surface differential reflectivity can be exploited for in situ monitoring of silver growth. ${ }^{12}$

The optical extinction spectra of metallic nanoparticles can be well described by the theory of Gustav Mie. ${ }^{13}$ However, this theory is rather limited to the spherical shape of the particles. For a qualitative description of effective media, it is possible to use a suitable theory, such as Maxwell-Garnett theory (MGT) and effective medium approximation. This theory is strictly limited to a particle size of $<0.1$ of the wavelength, but it can be generalized for arbitrary (nonspherical) shape using dynamical MGT. ${ }^{11,14}$

Besides the optical monitoring, a monitoring of the silver layer conductivity during the deposition process is a valuable tool for estimation of a percolation threshold, at which the electrical sheet resistance drops by several orders of magnitude. ${ }^{15}$

In this work, we study an ultrathin silver layer growth at the initial stage of growth. However, we also partially deal with silver oxide deposition. Silver oxide can exist in several phases, with $\mathrm{Ag}_{2} \mathrm{O}$ being the most thermodynamically stable. It decomposes at $>600 \mathrm{~K}$. Sputtering of a silver target in a reactive gas is a frequently used method for preparation of a silver oxide layer. ${ }^{16}$

\section{Experimental Details}

The silver ultrathin layers were deposited in a vacuum chamber by means of magnetron sputtering of silver target. The magnetron target was connected to a radiofrequency [(RF), 13.56 MHz)] generator. The RF power was set to $50 \mathrm{~W}$. The vacuum chamber was evacuated to an ultimate pressure of $2 \times 10^{-5} \mathrm{~Pa}$ before each experiment. The working pressure was set to $3 \mathrm{~Pa}$ by adjusting the Ar gas flow. The silver target surface was cleaned before each experiment by a presputtering process for $60 \mathrm{~s}$. The silver layers were grown on two types of fused silica substrates with dimensions of $14 \times 14 \mathrm{~mm}^{2}$. For optical transmission measurement, we used a substrate polished on both sides. The optical reflectivity measurement was performed on a substrate, which has the second side tarnished in order to avoid the unwanted reflection from the back side of the substrate. The distance from target to substrate was set $90 \mathrm{~mm}$. Some of the experiments were accomplished on predeposited silver oxide interlayer. The ultrathin $\mathrm{AgO}_{\mathrm{x}}$ layer was prepared by a sputtering process in the gas mixture consisting of argon and $30 \%$ oxygen. The deposition time was only $4 \mathrm{~s}$. Its mass thickness was estimated at $\sim 2 \mathrm{~nm}$.

The vacuum chamber is equipped with two oblique flanges with two fused silica windows. This arrangement allows in situ measurement of reflectivity of the substrate surface. Thus, the growth of the ultrathin layer can be monitored in real time. The optical reflectivity spectra were collected during the deposition process with a sampling rate of $10 \mathrm{~Hz}$. The optical spectra were measured by a spectrometer (USB4000, Ocean Optics) in the spectral range of $200-890 \mathrm{~nm}$. The light source (DH-2000, Ocean Optics) was coupled with a collimating lens by an optical fiber. A diameter of the collimated light beam near the sample was $\sim 1.5 \mathrm{~mm}$. The incident angle of the light beam was $76.5 \mathrm{deg}$. The reflected light was collected by a lens, which was coupled to the spectrometer by an optical fiber.

The electrical resistance of the layer was measured during the deposition process by means of a specialized apparatus. Two parallel silver electrodes were predeposited on a fused silica substrate. The width of the uncoated space between the electrodes was $10 \mathrm{~mm}$. The electrodes were connected to fast, precision multimeter (Agilent 34410A) by the four-wires method in order to minimize the influence of contact resistance to the measurement precision. The contacted electrodes were kept on a floating potential in order to minimize the effect of sample bias on the growth process. The electrical resistance was measured in the regime of constant current of 
$500 \mathrm{nA}$; nevertheless, the voltage was limited to $1 \mathrm{~V}$. The substrate holder was isolated from the grounded chamber wall.

The ellipsometric measurements were performed by means of a spectroscopic ellipsometer (J. A. Woollam) operating in the rotating analyzer mode. The principal ellipsometric angles $\psi$ and $\Delta$ were measured in a spectral range of $250-800 \mathrm{~nm}$ at incidence angles of 60 and $70 \mathrm{deg}$. The samples were analyzed by fitting the measured data to a parametrized model consisting of parallel layers on a semi-infinite substrate. In our model, we assumed smooth interfaces and homogeneous optical constants in order to simplify the model and minimize the number of fitted parameters. The surface roughness was considered as a top layer with optical constants determined by the effective media approximation theory (a mixture of voids and the underlying material).

\section{Results and Discussion}

We prepared a series of samples differing one from another by the layer thickness, which was given by different deposition time. The deposition rate of the silver growth was estimated from the thickness of Ag layer deposited in $84 \mathrm{~s}$. The thickness was estimated by simulation of transmission spectra of the Ag layer possessing the expected thickness around $50 \mathrm{~nm}$, see Fig. 1. At such thickness, the silver layer is still transparent; nevertheless, the thickness is high enough to minimize the fitting error due to surface roughness. The Ag layer thickness of this sample was estimated $54 \mathrm{~nm}$, resulting in a deposition rate of $0.64 \mathrm{~nm} \cdot \mathrm{s}^{-1}$.

The silver possesses typically low wetting to oxides substrates. Therefore, it has a tendency toward the Volmer-Weber growth mode, which is characterized by a noncontinual layer growth and island formation. The main stages of the silver layer growth are shown in scanning electron microscopy images in Fig. 2. Layers 5, 6, and 7 shown in Fig. 2(a) and 2(c) were prepared with a deposition time of 11.2, 12.8, and $16.8 \mathrm{~s}$, respectively. Assuming a constant deposition rate, we estimated the mass thickness of samples 5,6 , and 7 to be $7.2,8.3$, and $10.8 \mathrm{~nm}$, respectively. Figure 2(a) demonstrates the growth stage characterized by noncontinuous island formation and coalescence phenomena of the neighbor particles. The electrical resistance is beyond the measurable limit of the instrument ( $>1 \mathrm{G} \Omega$ ). The reflectivity spectrum of this sample is shown in Fig. 3(a). The reflectivity curve of the ideal continuous silver layer with corresponding mass thickness is shown in the same graph for comparison. The optical constants of Ag taken from literature ${ }^{17}$ were used for this simulation. The obvious discrepancy between these two curves in the graph is most pronounced in the visible and near-infrared region. This optical behavior is caused by a localized plasmon resonance in the silver nanoparticles.

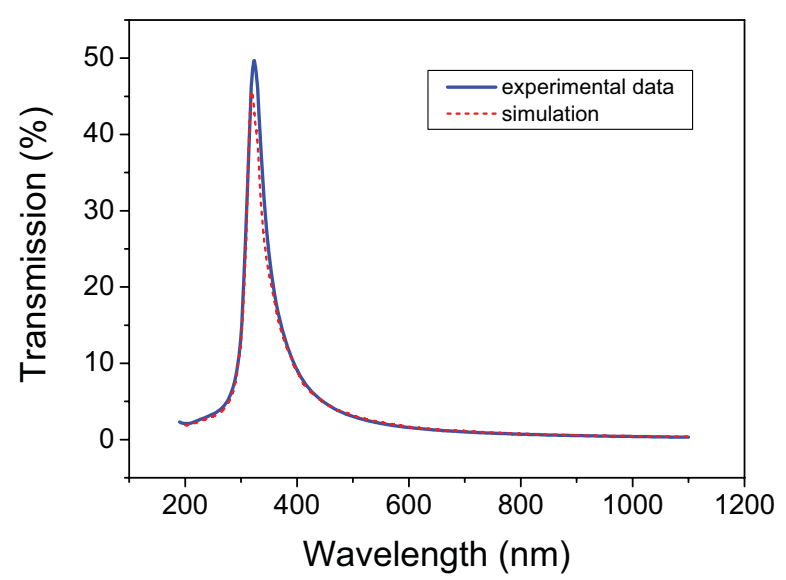

Fig. 1 Transmission spectrum of the Ag layer on glass substrate. The dashed curve shows a simulated transmission spectrum for ideal silver layer with a thickness of $54 \mathrm{~nm}$. 

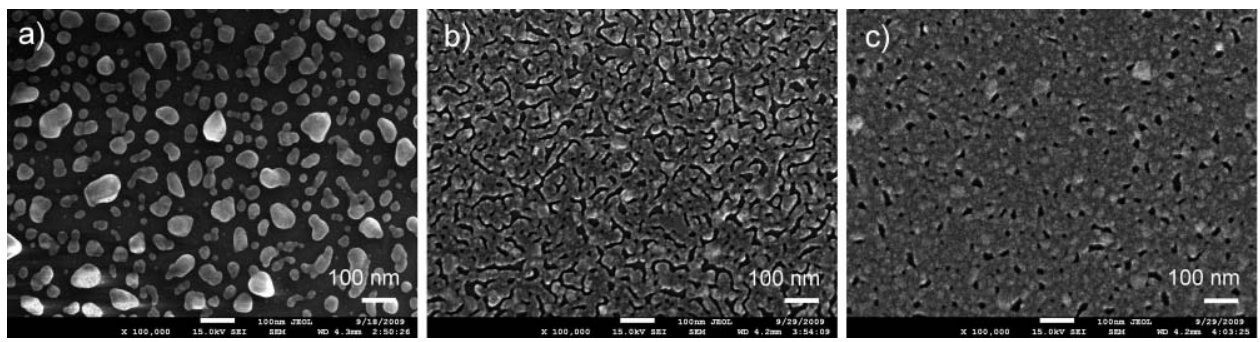

Fig. 2 SEM photograph of three different ultrathin silver layers grown on fused silica substrate. The micrographs represent different stages of the growth: (a) 11.2, (b) 12.8, and (c) $16.8 \mathrm{~s}$.

The growth stage beyond the percolation threshold is represented by sample 6 , which is shown in Fig. 2(b). The morphology of this layer is still highly disordered, but its electrical resistance, reaching $142 \Omega$, dropped by several orders of magnitude compared to that of the previous sample. There are long disordered holes seen in the scanning electron microscope (SEM) image that are represented as wormlike dark spots. Nevertheless, the silver coating (gray) already exhibits certain connectivity, which is confirmed by measurement of the electrical resistance. The reflectivity spectrum of this sample shows a significant localized plasmon resonance effect [see Fig. 3(b)].

The SEM image in Fig. 2(c) shows a nearly continuous layer with some remaining holes represented as dark spots. The electrical resistance of this sample is $35 \Omega$. The reflectivity spectrum of this sample shows a nearly similar behavior to the simulation, as shown in Fig. 3(c). As a result of this experiment, we can conclude that comparative analysis of the sample reflectivity can indicate the morphology of the silver layer. Thus, we can identify the growth mode of the deposition process. Therefore, it would be useful to use a real-time monitoring of the reflectivity during the deposition process for identification of the growth mode. The SEM images demonstrate that the morphology of the Ag ultrathin layers significantly change during a few seconds of the deposition process. Accurate control of the growth would be difficult without appropriate monitoring.

The optical properties of the prepared samples were analyzed by spectral ellipsometry measurement in the spectral range from 250 to $800 \mathrm{~nm}$, at two incidence angles of 60 and 70 deg. The graph of ellipsometric angles $\psi$ and $\Delta$ as functions of photon energy is shown in Fig. 4. These data were fitted using a model of layered structure fused silica/silver layer/air ambient, where the dispersion of the silver layer was parametrized using a model of several Cody-Lorentz oscillators. The roughness of sample 7 was included in the model as a top layer with optical constants determined by effective media approximation theory. The simulation curve fits the measured data of $\psi$ and $\Delta$ of all the samples well, as shown in Fig. 4 . The fitting
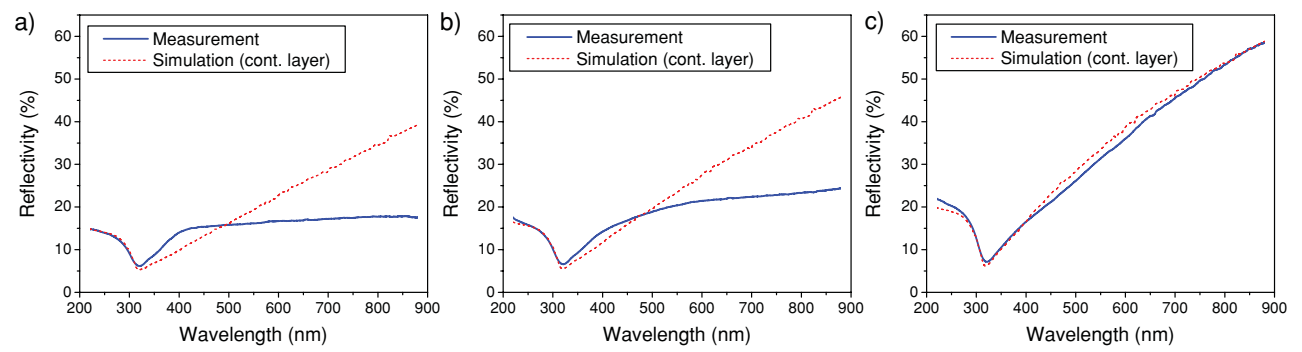

Fig. 3 Reflectivity spectra of three different ultrathin silver layers grown on fused silica substrate. The reflectivity was measured at near-to-normal angle of incidence. The samples were prepared at different stages of the growth: (a) 11.2, (b) 12.8, and (c) $16.8 \mathrm{~s}$. The dashed curve shows a simulation of ideal continuous silver layer with its thickness corresponding to mass thickness of the experimentally prepared layer. 

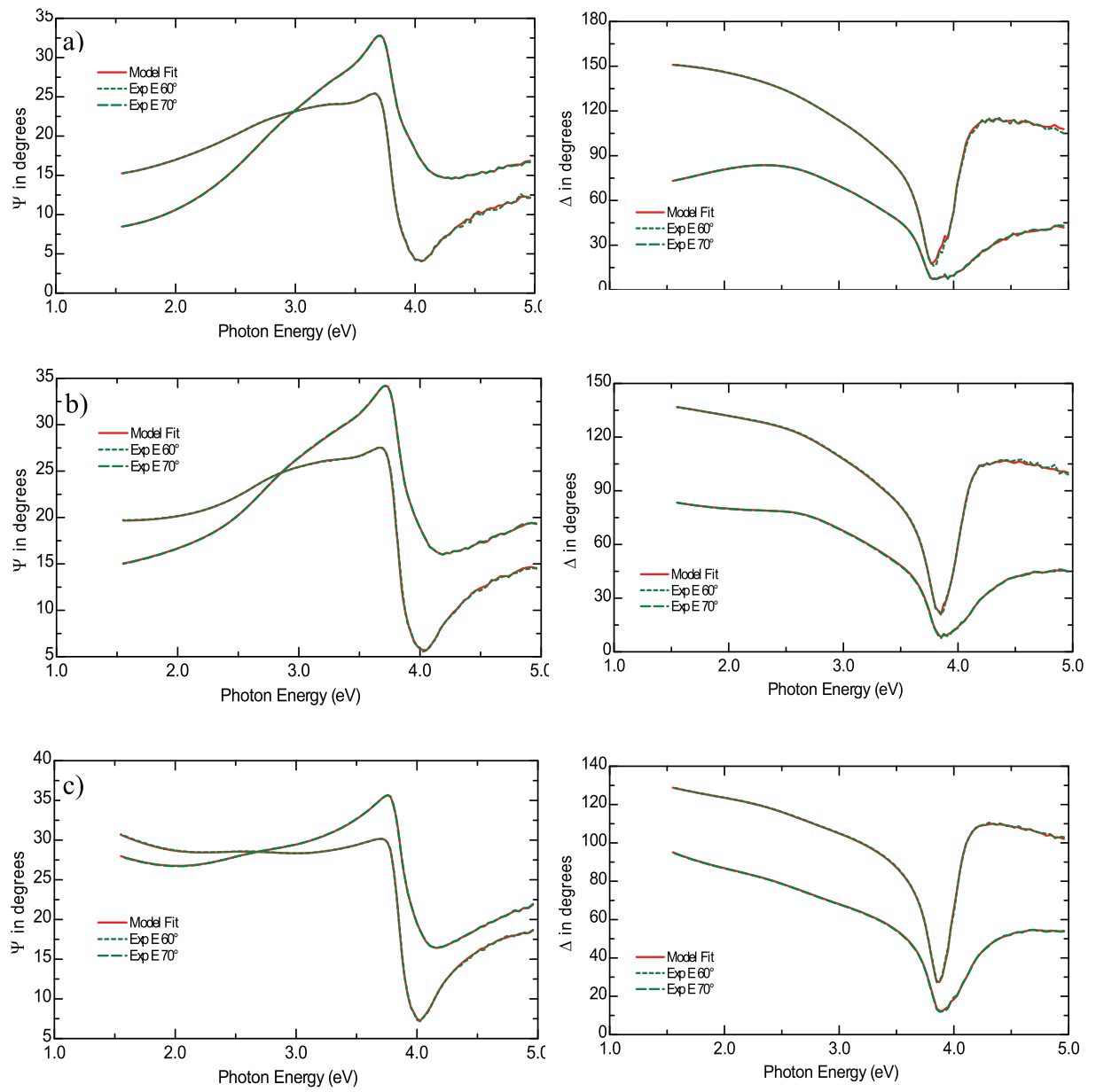

Fig. 4 Experimentally obtained ellipsometric angles $\psi$ and $\Delta$ as the function of photon energy at the incidence angles of 60 and 70 deg for samples: (a) 05, (b) 06, and (c) 07 . The straight line corresponds to a simulation.

procedure yielded thickness values of $9.2 \mathrm{~nm}$ for sample $5,11.6 \mathrm{~nm}$ for 6 , and $10.6+3.6 \mathrm{~nm}$ of roughness for sample 7 . These thickness values are higher than those of estimated effective mass thickness; however, this result is consistent with the fact that mass thickness describes a solid silver layer with a high packing density, whereas the studied ultrathin silver films exhibit certain porosity.

The resulting index of refraction and extinction coefficient of the ultrathin silver films is shown in Fig. 5. The optical constants of the bulk silver (obtained from literature ${ }^{17}$ ) are plotted in the same graph for comparison. It can be seen that sample 7 exhibits optical constants nearest to those of the bulk silver. The noncontinuous silver layers (samples 5 and 6) exhibit a gradual increase of the refractive index for electron energy below $3 \mathrm{eV}$ with decreasing layer thickness. In the same spectral range, the extinction coefficient exhibits lower values than that of bulk silver.

The percolation threshold is very often defined as the point of growth at which the metallic layer becomes electrically conductive. We set up an experiment of real-time monitoring of the electrical resistance of the grown Ag layer. The layer was grown at the same deposition conditions as in the previous experiments. Nevertheless, the growth rate rather decreased due to shadowing effect of the apparatus for electrical connection. The deposition time was $60 \mathrm{~s}$. 


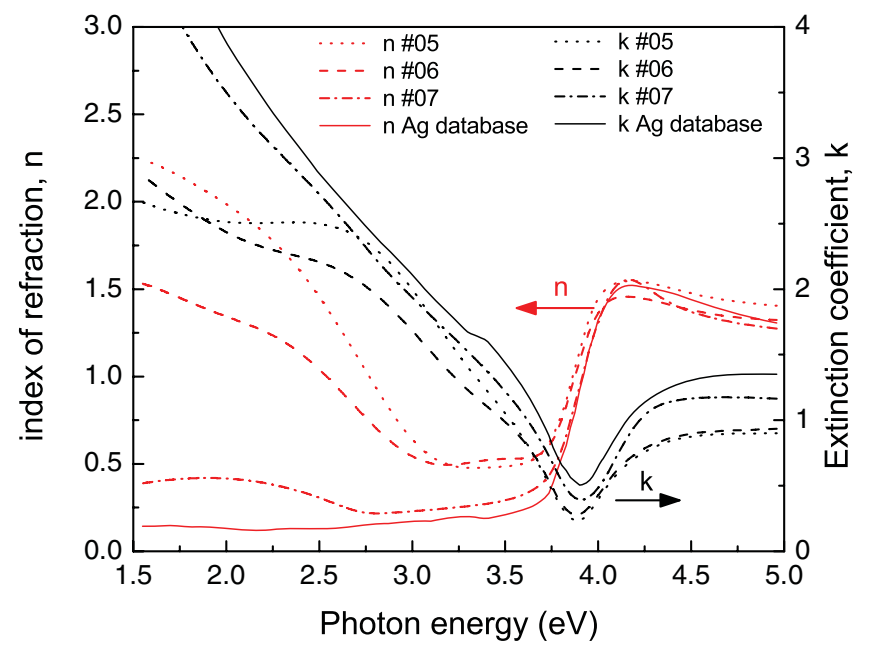

Fig. 5 Optical constants of ultrathin Ag layers prepared with deposition time of 11.2, 12.8, and $16.8 \mathrm{~s}$, respectively.

The complete layer thickness was estimated at $24 \mathrm{~nm}$, giving the growth rate $0.4 \mathrm{~nm} / \mathrm{s}$. The development of the electrical resistance is shown in Fig. 6. At the beginning, the resistance is beyond the measurement limit, which is affected by plasma discharge conductivity. At a certain time point of the deposition process, the electrical resistance drops down by several orders of magnitude, indicating the percolation threshold. This threshold was indicated in this experiment at deposition time $37.5 \mathrm{~s}$. After that, the electrical resistance decrease reaching value of $2.2 \Omega$ for the completed layer. The resulting electrical resistivity was estimated $7.4 \times 10^{-8} \Omega \mathrm{m}$. This value is higher than that of bulk silver due to scattering of conduction electrons at films surfaces. The silver-layer growth was monitored by means of an optical reflectance of the coated layer, which was measured simultaneously with the electrical resistance monitoring. The evolution of the silver-layer reflectance during the deposition process is shown in Fig. 7. The sample reflectivity at the percolation threshold is shown by a dotted curve. This highlighted curve exhibits a resonant feature, which is most pronounced toward the infrared part of the spectrum. At this part, the measured reflectivity significantly differs from the simulated reflectivity of the continuous silver layer of the equivalent thickness. This simulation is plotted in this graph as a

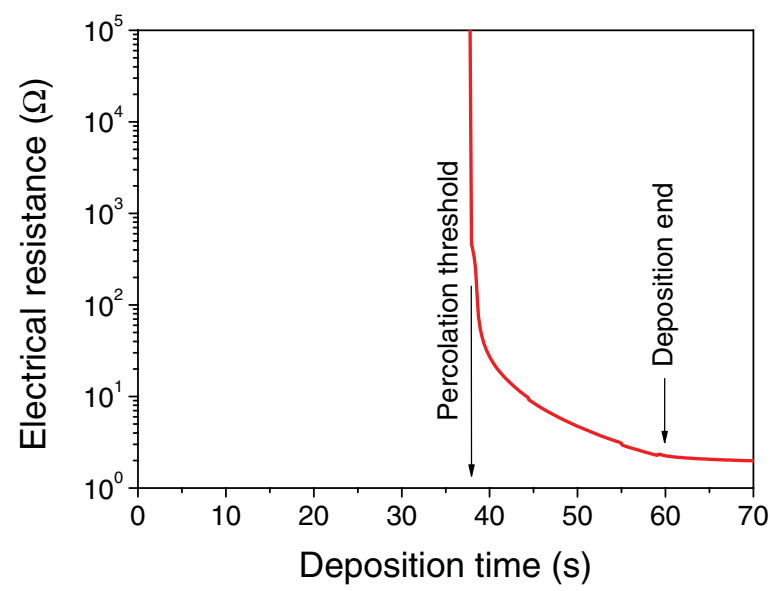

Fig. 6 Real-time monitoring of electrical resistance during the deposition process of the silver layer growth. 


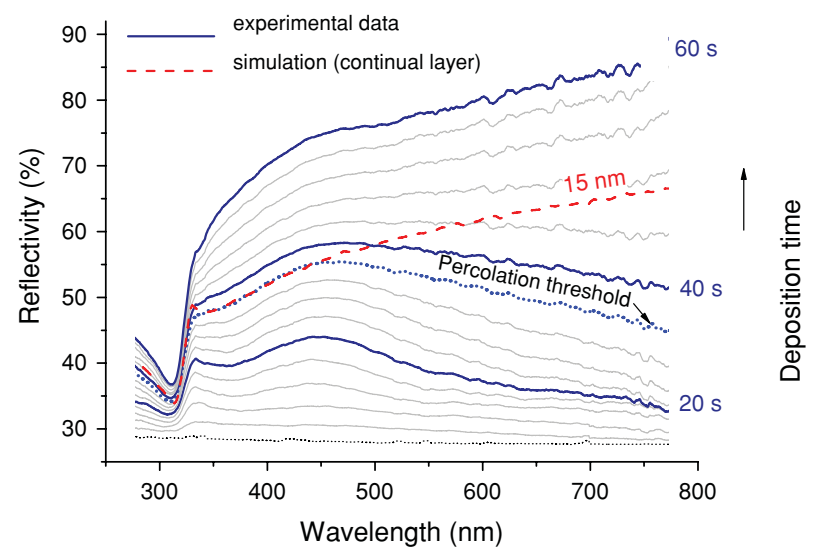

Fig. 7 Real-time monitoring of reflectivity spectra during the deposition process of silver layer.

dashed curve. From this graph, it is obvious that the localized plasmon resonance occurs even at the percolation threshold. The localized plasmon resonance is induced by collective oscillations of free electrons, which are confined within the silver grain. ${ }^{13}$ From the experiment described above, we can conclude that this plasmon resonance can exist even after interconnection of the silver grains, forming a nanostructured silver layer similar to that shown in Fig. 2(b). This resonant character disappears at a deposition time of $\sim 50 \mathrm{~s}$, when the nanostructuring of the silver film disappears (see Fig. 7).

In the discussion above, we have shown that the optical monitoring can be used for identification of the growth mode, which can be influenced by wetting properties of silver to substrate. This behavior is dependent on the substrate material. In the following experiment, we assume that silver possesses good wetting properties to its own oxides.

In a comparative experiment, we deposited two ultrathin silver layers. The growth was monitored by real-time measurement of the coating's reflectivity during the deposition process. In the first experiment, the silver layer was deposited on the bare fused silica substrate. The estimated mass thickness of the completed layer was $9.6 \mathrm{~nm}$. Figure 8(a) shows the evolution of the measured reflectivity spectra during the deposition process. The dotted curves exhibit a simulation of the reflectivity of an ideal continuous silver layer. It is obvious that the discrepancy between the measured and simulated curves indicates noncontinuous silver-layer growth typical
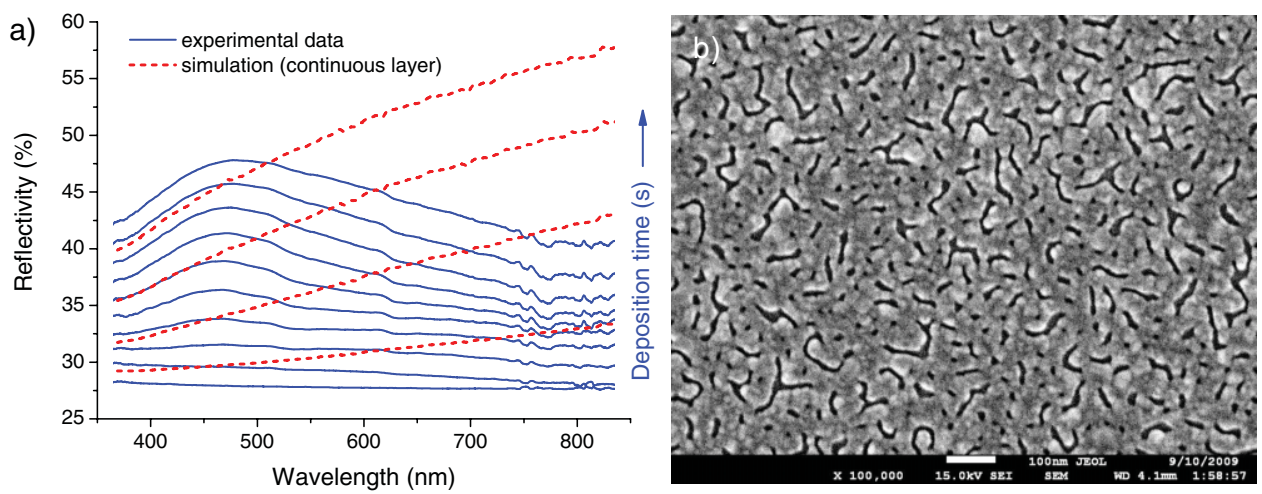

Fig. 8 (a) Real-time monitoring of reflectivity spectra during the deposition process of the ultrathin silver layer and (b) SEM image of the completed Ag layer. 

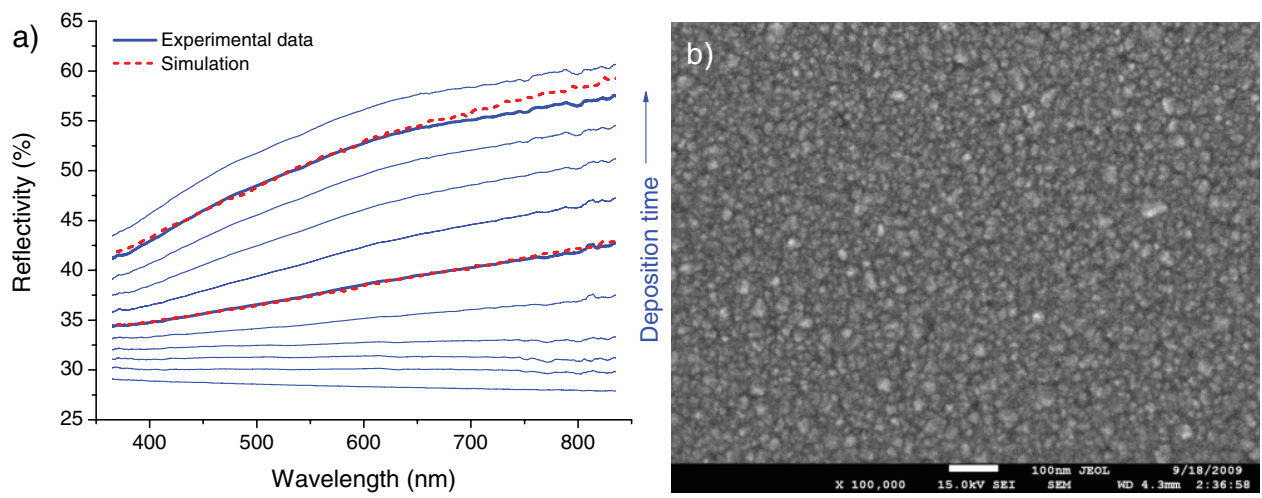

Fig. 9 (a) Real-time monitoring of reflectivity spectra during the deposition process of the ultrathin silver layer and (b) SEM image of the completed Ag layer. The growth mode was influenced by $\mathrm{AgO}_{\mathrm{x}}$ coating prepared on the substrate before the deposition.

for Volmer-Weber growth mode. This observation is confirmed by the SEM image of the completed silver layer that is shown in Fig. 8(b).

The second experiment was accomplished on a fused silica substrate coated with an ultrathin silver oxide layer $(\sim 2 \mathrm{~nm})$, which was predeposited on the substrate before the silver deposition experiment. The silver layer was deposited over the $\mathrm{AgO}_{\mathrm{x}}$ interlayer. The monitored reflectivity spectra are shown in Fig. 9(a). The simulation of the continuous Ag layer fits the measured spectra well. The resonant behavior is not observed in the measured spectra. This indicates the continuous silver layer growth since the initial stage of the deposition process. The SEM image in Fig. 9(b), showing a continuous silver layer confirms this observation. The estimated mass thickness was $11.3 \mathrm{~nm}$. The roughness of this layer, estimated by atomic force microscopy, was $1.8 \mathrm{~nm}$ (rms). We expect that the $\mathrm{AgO}_{\mathrm{x}}$ interlayer can decompose during the early stage of the silver-layer growth due to low stability of the silver oxide. The subsequent silver growth is characterized by a strong adatom-to-surface interaction, which lead to a continuous silver-layer formation.

\section{Conclusion}

In this work, we proved that optical monitoring is a valuable tool to identify the growth mode in real time during the deposition process. The percolation threshold can be determined by electrical resistance monitoring. The continuous silver-layer growth is indicated by means of monitoring of the reflectivity spectra during the deposition. The growth mode of the silver can be influenced by predeposited $\mathrm{AgO}_{\mathrm{x}}$ interlayer. Thus, we can prepare a continuous ultrathin silver layer.

\section{Acknowledgments}

This research was supported by Grant Agency of Czech Academy of Sciences, Projects Nos. IAA100100718, AVOZ 10100522, and KAN 400100653; and Grant Agency of the Czech Republic, Project No. GACR 202/09/P324. One of the coauthors thanks the VAKUUM Praha for partial support of his research. The authors thank M. Škereň for SEM analysis, M. Bodnár for the monitored data processing, and V. Kulykovskyy for a fruitful discussion.

\section{References}

1. A. Piegari and J. Bulír, "Variable narrow-band transmission filters with wide rejection band for spectrometry," Appl. Opt. 45, 3768-3773 (2006). 
2. A. Piegari, J. Bulír, and A. Krasilnikova Sytchkova, "Variable narrow-band transmission filters for spectrometry from space. 2. Fabrication process," Appl. Opt. 47, C151-C156 (2008).

3. F. Sarto, M. S. Sarto, M. C. Larciprete, and C. Sibilia, "Transparent films for electromagnetic shielding of plastics," Rev. Adv. Mater. Sci. 5, 329-336 (2003).

4. C. T. Campbell, "Ultrathin metal films and particles on oxide surfaces: Structural, electronic and chemisorptive properties," Surf. Sci. Rep. 27, 1-111 (1997).

5. N. Doraiswamy, G. Jayaram, and L. D. Marks, "Unusual island structures in Ag growth on Si(100)-(2×1)," Phys. Rev. B 51, 10167-10170 (1995).

6. C. Wen, K. Ishikawa, M. Kishima, and K. Yamada, "Effects of silver particles on the photovoltaic properties of dye-sensitized $\mathrm{TiO}_{2}$ thin films," Sol. Energy Mater. Sol. Cells 61, 339-351 (2000).

7. D. M. Schaadt, B. Feng, and E. T. Yu, "Enhanced semiconductor optical absorption via surface plasmon excitation in metal nanoparticles," Appl. Phys. Lett. 86, 063106 (2005).

8. K. R. Catchpole and S. Pillai, "Absorption enhancement due to scattering by dipoles into silicon waveguides," J. Appl. Phys. 100, 044504 (2006).

9. K.-C. Lee, S.-J. Lin, C.-H. Lin, C.-S. Tsai, and Y.-J. Lu, "Size effect of Ag nanoparticles on surface plasmon resonance," Surf. Coat. Technol. 202, 5339-5342 (2008).

10. G. Xu, M. Tazawa, P. Jin, and S. Nakao, "Surface plasmon resonance of sputtered Ag films: Substrate and mass thickness dependence," Appl. Phys. A 80, 1535-1540 (2005).

11. S. K. Mandal, R. K. Roy, and A. K. Pal, "Effect of particle shape distribution on the surface plasmon resonance of $\mathrm{Ag}-\mathrm{SiO}_{2}$ nanocomposite thin films," J. Phys. D 36, 261-265 (2003).

12. R. Lazzari and J. Jupille, "Silver layers on oxide surfaces: Morphology and optical properties," Surf. Sci. 482-485, 823-828 (2001).

13. U. Kreibich and M. Vollmer, Optical Properties of Metal Clusters, Springer-Verlag, Berlin (1995).

14. Y. M. Wu, L. Gao, and Z. Y. Li, "The influence of particle shape on nonlinear optical properties of metal-dielectric composites," Phys. Stat. Sol. B 220, 997-1008 (2000).

15. K. Fukuda, Sunnit H. N. Lim, and A. Anders, "Coalesce of magnetron-sputtered silver islands affected by transition metal seeding ( $\mathrm{Ni}, \mathrm{Cr}, \mathrm{Nb}, \mathrm{Zr}, \mathrm{Mo}, \mathrm{W}, \mathrm{Ta})$ and other parameters," Thin Solid Films 516, 4546-4552 (2008).

16. R. Snyders, M. Wautelet, R. Gouttebaron, J. P. Dauchot, and M. Hecq, "Experimental and theoretical studies of the DC reactive magnetron sputtering deposition of silver oxide thin films," Surf. Coat. Technol. 174-175, 1282-1286 (2003).

17. E. D. Palik, Handbook of Optical Constants of Solids I, Academic Press, New York (1985).

Jiř́ Bulír has been employed at Institute of Physics of the Academy of Sciences since 1992. He received his $\mathrm{PhD}$ from Czech Technical University in Prague, 1997. He participated on a project for development of metal-dielectric linearly variable filters during his stays at the Research Center ENEA (Optical Coatings Group). His recent research focuses on optical behavior of nanostructured metallic layers, and his expertise is in layer growth processes and physical vapor deposition methods. He is the author and coauthor of more than 36 journal papers. He is a member of SPIE.

Michal Novotný is a postdoctoral fellow at the Institute of Physics. He received his MS and $\mathrm{PhD}$ in physics from the Czech Technical University in Prague in 2001 and 2006, respectively. $\mathrm{He}$ is the author and coauthor of more than 30 journal papers. His current research interests include thin-film deposition by PVD techniques, thin-film properties in situ characterization, and plasma spectroscopy. He is a member of SPIE.

Anna Lynnykova is a PhD student at the Czech Technical University in Prague. She received her MS in biophysics from the Donetsk National University in 2002. She is a coauthor of about 10 journal papers. Her current research interests include thermo-optical ellipsometry measurements of perovskite thin films and crystals. 
Ján Lančok is a senior researcher at the Institute of Physics of the Academy of Science of the Czech Republic. He received his MS in physics from the Czech Technical University of Prague in 1996 and his PhD in physical engineering from Faculty of Nuclear Sciences and Physical Engineering at Czech Technical University of Prague. He is the author and coauthor of more than 50 journal papers. His current research interests are focused on the deposition of thin films by physical vapor deposition techniques. 